\title{
Functional brain imaging study of mathematical reasoning abilities in velocardiofacial syndrome (del22q11.2)
}

Stephan Eliez, MD, Christine M. Blasey, PhD, Vinod Menon, PhD, Christopher D. White, BA, J. Eric Schmitt, BS, and Allan L. Reiss, MD

\begin{abstract}
Purpose: Children with velocardiofacial syndrome (VCFS) often have deficits in mathematical reasoning. Previous research has suggested that structural abnormalities in the parietal lobe region might underlie these deficits. The present study utilized functional magnetic resonance imaging (fMRI) to explore the relationship between brain function and mathematical performance in VCFS. Methods: Eight children with VCFS and eight comparison subjects underwent fMRI scanning and completed an arithmetic computation task. Results: In the VCFS group, increased activation was observed in the left supramarginal gyrus (LSMG) as the task difficulty increased. Conclusion: Aberrant LSMG activation, possibly due to structural deficits of the left parietal lobe, may explain decrements in arithmetic performance observed in VCFS. Genetics in Medicine, 2001:3(1):49-55.
\end{abstract}

Key Words: velocardiofacial syndrome, deletion 22q11, brain, functional MRI, mathematical reasoning

Persons with velocardiofacial syndrome (VCFS) have cognitive deficits and typically score in the borderline to mildly mentally retarded range on standardized tests of general cognitive ability. ${ }^{1-5}$ In addition to general cognitive disability, children with VCFS also show more specific deficiencies in the area of mathematics ability.

The first descriptive study of the cognitive phenotype associated with VCFS ${ }^{2}$ showed that children with this condition scored disproportionately lower on math and reading comprehension tests relative to tests of language. For example, an early study demonstrated that math scores from the Wide Range Achievement Test (WRAT) among 6-11 year olds with VCFS ranged from 81 to 90 (population mean $=100, \mathrm{SD}=10$ ) and from 74 to 86 among affected adolescents. ${ }^{2}$ More recent investigations have yielded similar results. ${ }^{3,6,7}$ General weaknesses in problem solving and manipulation of numbers have been observed among school-age children with VCFS, as well as particular difficulties in converting language into number sentences, solving problems with decimals and fractions, telling time, and converting money. ${ }^{6}$ When examining standardized test scores in clinical samples of 33 subjects with VCFS, Moss et al. ${ }^{3}$ reported lower composite math achievement scores relative to composite scores of reading and spelling. In accordance with these findings, Swillen et al. ${ }^{7}$ recently reported that children with VCFS had significant decrements in arithmetic per-

\footnotetext{
From the Department of Psychiatry and Behavioral Sciences, Stanford University School of Medicine, Stanford, California.

Stephan Eliez, MD, Department of Psychiatry and Behavioral Sciences, Stanford University School of Medicine, 401 Quarry Road, Stanford, CA 94305-5719.

Received: July 27, 2000.

Accepted: October 24, 2000.
}

formance alongside relatively preserved abilities in reading and spelling.

Although the neural substrates that underlie deficiencies in arithmetic reasoning in VCFS are currently unknown, research focused on individuals with neurological dysfunction from other causes has begun to define the specific brain regions that are involved in mathematical performance. For example, human brain lesion studies have demonstrated acalculia among patients with lesions to the parietal cortex. Further, the parietal area is thought to underlie the acalculia component of Gerstmann's syndrome. ${ }^{8-13}$

Functional magnetic resonance imaging (fMRI) studies with "normal" subjects also have implicated several brain regions as being associated with arithmetic performance. ${ }^{14,15}$ During "approximate" computation, brain activation has been observed in the bilateral inferior parietal lobule, right precuneus, bilateral precentral sulci, left dorsolateral prefrontal cortex, left superior prefrontal gyrus, left cerebellum, and left and right thalami. ${ }^{15}$ During "exact" computation, activation has been reported in left and right prefrontal regions, left cingulate gyrus, left precuneus, right parieto-occipital sulcus, left and right angular gyrus, and right middle temporal gyrus. ${ }^{16}$ Despite considerable variability in regional patterns of activation, these imaging studies collectively suggest that both prefrontal and parietal cortices are involved in arithmetic tasks.

Evidence of parietal lobe involvement in mathematics is of particular interest to VCFS research, given that structural aberrations in the parietal region have been observed in affected children. ${ }^{17}$ Specifically, reduced parietal lobe volume, particularly in the left hemisphere, coupled with relative preservation of frontal lobe volume, was reported in a recent quantitative morphological investigation of children and adolescents with VCFS. ${ }^{17}$ 
The purpose of the current study was to explore parietal lobe function in children with VCFS and to consider whether abnormal patterns of activation in this region were associated with math deficits commonly observed among individuals with this disorder. We hypothesized that math performance would be significantly lower in the VCFS group compared to controls; further, we anticipated that fMRI would illustrate group differences in the activity of the parietal lobe during a math performance task.

\section{METHODS}

\section{Subjects}

Sixteen $(N=16)$ subjects participated in the study. Eight persons with VCFS ( 3 females and 5 males; mean age $15.5 \pm$ 3.6 years) and eight matched controls ( 3 females and 5 males; mean age $15.8 \pm 4.1$ years) underwent behavioral testing and fMRI. Persons with VCFS were recruited from the Northern California VCFS organization and through our Web site (www-cap.stanford.edu). Only subjects with VCFS who were diagnosed with a 22q11.2 microdeletion confirmed by the fluorescence in situ hybridization (FISH) technique were included in the study. All subjects were identified as having the typical "large" $3 \mathrm{Mb}$ deletion. ${ }^{18}$ The deletions were verified and their extent determined by two-color FISH, with cosmid probes DO832 (COMT) and N48C12 (D22S264), as described. ${ }^{19}$ The two probes are specific for the proximal and distal deletion regions, respectively. ${ }^{20}$ Comparison group subjects were recruited locally through advertisements. Right handedness and absence of previous neurological, learning, or psychiatric disorder were used as an inclusion criteria for control subjects. VCFS participants who were left-handed were not excluded, given that loss of hemispheric specialization may be part of the expressed phenotype. After providing a complete description of the study to all potential participants, written informed consent was obtained under protocols approved by the Institutional Review Board of Stanford University.

\section{fMRI experimental design: arithmetic computation}

This experiment began with a 30-second rest epoch followed by six alternating 30 -second epochs of "easy" (2-operand equation) experimental and control trials. The six "easy" epochs were followed by a second 30-second rest epoch. After the second rest epoch, six alternating 30-second epochs of "difficult" (3-operand equation) experimental and control trials were presented. Following these six difficult epochs was a third 30 -second rest epoch. During the rest condition, subjects passively viewed a blank screen. Easy experimental epochs consisted of five, 2-operand addition or subtraction problems (randomly intermixed) with either a correct or an incorrect resultant (e.g., $1+2=3$ or $5-2=4$ ). Difficult experimental epochs consisted of five, 3-operand addition/subtraction problems with either a correct or an incorrect resultant (e.g., 6 $-3+5=8$ or $6+2-3=4$ ). All experimental and control stimuli were presented for $5250 \mathrm{msec}$, with an interstimulus interval (ISI) of $750 \mathrm{msec}$. Half of the results were correct and required a button press, and the other half were incorrect. Of the incorrect-resultant trials, half of the results were one more than the correct answer, and half were one less than the correct answer. During all experimental epochs the instructions "Push if Correct" were displayed for the entire length of the epoch. Subjects were instructed to respond with a key press only when the resultant of the math equation was correct. Control epochs for the blocks of "easy" experimental trials were composed of five stimuli, each consisting of a string of five single digits. Control epochs for the blocks of "difficult" experimental trials were composed of five stimuli, each consisting of a string of seven single digits. During the control epochs, the instruction "Push for 0" was displayed throughout the entire length of the epoch $(6000 \mathrm{msec})$. Specifically, subjects were instructed to respond with a key press only when a zero appeared in the string of digits.

\section{fMRI procedures}

\section{Acquisition}

Images were acquired on a $1.5 \mathrm{~T}$ GE Signa scanner with Echospeed gradients using a custom-built whole head coil that provides a $50 \%$ advantage in signal-to-noise ratio over that of the standard GE coil. ${ }^{21} \mathrm{~A}$ custom-built head holder was used to prevent head movement. Eighteen axial slices $(6 \mathrm{~mm}$ thick, 1 $\mathrm{mm}$ skip) parallel to the anterior and posterior commissure covering the whole brain were imaged with a temporal resolution of 2 seconds using a $2^{\star}$ weighted gradient echo spiral pulse sequence $(\mathrm{TR}=2000 \mathrm{msec}, \mathrm{TE}=40 \mathrm{msec}$, flip angle $=$ $89^{\circ}$ and 1 interleave). ${ }^{22}$ The field of view was $240 \mathrm{~mm}$, and the effective in-plane spatial resolution was $4.35 \mathrm{~mm}$. To aid in localization of functional data, high resolution T1 weighted spoiled grass gradient recalled (SPGR) 3D MRI sequence with the following parameters was used: $\mathrm{TR}=24 \mathrm{msec}$; $\mathrm{TE}=5$ msec; flip angle $=40^{\circ} ; 24 \mathrm{~cm}$ field of view; 124 slices in sagittal plane; $256 \times 192$ matrix; acquired resolution $=1.5 \times 0.9 \times 1.2$ $\mathrm{mm}$. The images were reconstructed as a $124 \times 256 \times 256$ matrix with a $1.5 \times 0.9 \times 0.9 \mathrm{~mm}$ spatial resolution. Structural and functional images were acquired in the same scan session.

The task was programmed using Psyscope ${ }^{23}$ on a Macintosh (Sunnyvale, CA) notebook computer. Initiation of scan and task was synchronized using a TTL pulse delivered to the scanner timing microprocessor board from a "CMU Button Box" microprocessor (http://poppy.psy.cmu.edu/psyscope) connected to the Macintosh. Stimuli were presented visually at the center of a screen using a custom-built magnet compatible projection system (Resonance Technology, CA).

\section{Image preprocessing}

Images were reconstructed, by inverse Fourier transform, for each of the 225 time points into $64 \times 64 \times 18$ image matrices (voxel size: $3.75 \times 3.75 \times 7 \mathrm{~mm}$ ). The fMRI data were preprocessed using SPM99 (http://www.fil.ion.ucl.ac.uk/ $\mathrm{spm}$ ). Images were corrected for movement using least square minimization without higher-order corrections for spin history, and normalized to stereotaxic Talairach coordinates. ${ }^{24}$ 
Images were then re-sampled every $2 \mathrm{~mm}$ using sinc interpolation and smoothed with a $4 \mathrm{~mm}$ Gaussian kernel to decrease spatial noise.

\section{Region of interest (ROI) image processing}

\section{Voxel-based}

Group analysis was performed using a random-effects model that incorporated a two-stage hierarchical procedure. This model estimates the error variance for each condition of interest across subjects, rather than across scans ${ }^{25}$ and, therefore, provides a stronger generalization to the population from which data are acquired. This analysis proceeded in two steps. In the first step, contrast images for each subject and each effect of interest were generated as described above. In the second step, these contrast images were analyzed using a general linear model to determine voxel-wise t-statistics. One contrast image was generated per subject, per effect of interest (e.g., 3 seconds ISI, 2-operand condition, minus the control condition). A one-way t-test was then used to determine group activation for each effect. Finally, the t-statistics were normalized to Z scores, and significant clusters of activation were determined using the joint expected probability distribution of height and extent of $\mathrm{Z}$ scores, ${ }^{26}$ with height $(\mathrm{Z}>2.33 ; P<0.01)$ and extent thresholds $(P<0.05)$.

\section{Group analysis: ROI-based}

The aim of this analysis was to investigate the profile of regional task-related differences in activation. Group analysis was conducted using $\mathrm{Z}$ scores derived from the individual subject analysis as described above. The percentage of voxels, in the left SMG was determined and entered into a repeated measures analysis of variance (ANOVA) for the two task conditions (easy, difficult). An alpha-level for significance of $P<$ 0.05 (two-tailed) was used.

\section{Definition of ROls}

ROIs (see Fig. 1) were demarcated separately in the left and right hemispheres from the average T1-weighted Talairach normalized images.

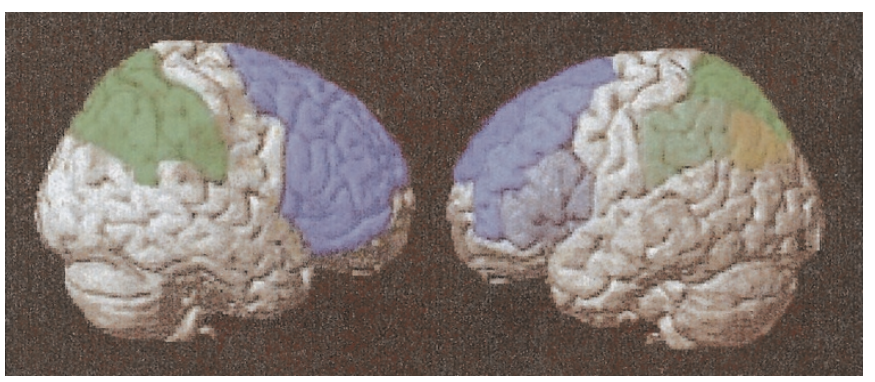

Fig. 1 This rendered surface illustration shows how the frontal (blue) and parietal (green) lobes (left side image) are divided (right side image) into superior frontal (dark blue), middle frontal (medium blue), inferior frontal (light blue), superior parietal (dark green), supramarginal (light green), and angular (yellow) gyri.

\section{Inferior frontal gyrus}

Using a surface rendering of the average brain, the anterior border of the inferior frontal gyrus (IFG) was identified by the junction of the lateral orbital sulcus and inferior frontal sulcus. The posterior border was defined by the precentral sulcus. The superior border was defined by the inferior frontal sulcus. The inferior border was defined by the lateral orbital sulcus until it could no longer be visualized. For the most posterior part of the IFG, the inferior border was the horizontal ramus of the lateral fissure. Medially, the border was defined by a straight line joining the deepest point of the inferior frontal sulci with the deepest point of the lateral orbital sulci. These definitions closely followed surface sulci demarcated by Duvernoy and Bourgouin $^{27}$ and Ono et al. ${ }^{28}$

\section{Middle frontal gyrus}

The superior frontal sulcus defined the medial boundary for the middle frontal gyrus (MFG). The frontomarginal sulcus and the inferior frontal sulcus defined the lateral boundary. At more posterior slices where the inferior frontal sulcus disappears, the superior precentral sulcus was used as the lateral boundary. The posterior boundary for this region was the precentral gyrus.

\section{Superior frontal gyrus}

The superior-medial border of the superior frontal gyrus (SFG) was defined as the cingulate sulcus. The superior precentral sulcus was used as the posterior border. Finally, the superior frontal sulcus was followed until it curves medially toward the interhemispheric fissure to define the lateroinferior and anterior border of the SFG.

\section{Parietal cortex}

Information from multiplanar views was used to delineate the parietal lobe, and parcel the lobe into superior parietal lobule (SPL), supramarginal gyrus (SMG), and angular gyrus (ANG). The parietal lobe was delineated by first marking the postcentral sulcus as its anterior border. The inferior border was the Sylvian fissure until it was no longer identifiable in the coronal section progressing in the anterior to posterior direction. At this point, the inferior and medial borders were defined by the parallel sulcus and the interhemispheric fissure, respectively. Next, in the mid-sagittal view, a line parallel to the parieto-occipital fissure was identified and linearly interpolated to the parieto-occipital notch on the lateral surface. This defined the posterior border of the parietal lobe. The parietal lobe was then divided using neuroanatomical landmarks identified in the axial plane. The intraparietal sulcus was used to demarcate the SPL from the SMG and ANG. The intraparietal sulcus and the ascending posterior segment of the parallel sulcus were then used to separate the SMG and ANG.

\section{Statistical analyses}

Based on a general linear model, analyses of variance (ANOVA) were used to compare group means on the number 
of correct responses, reaction time, percentage of voxels activated (ROI-based). ANOVA was also used to compare activation levels in the ROIs. For regions showing activation differences, repeated-measures ANOVA was utilized to detect a pattern of group differences in the "easy" versus "difficult" math conditions. An alpha of 0.05 (two-tailed) was used as the threshold for determining statistical significance.

\section{RESULTS}

\section{Math performance}

As shown in Table 1, the average number of correct responses on the entire math task, including both the easy and difficult components, was significantly lower for VCFS subjects compared to controls (VCFS: 82.9\% \pm 14 ; Controls: $97.1 \% \pm 4 ; \mathrm{F}=7.5, \mathrm{df}=1,14, P=0.016)$. Accuracy was lower for VCFS subjects on the "easy" math questions, although this difference was not statistically significant $(\mathrm{F}=3.2, \mathrm{df}=1,14, P$ $=0.094)$. During the "difficult" condition, which composed more difficult math questions (see Materials and Methods section), a significant group difference in the average number of correct response was observed; the VCFS group performed worse relative to the controls $(\mathrm{F}=7.8, \mathrm{df}=1,14, P=0.014)$. Comparisons of mean reaction time during the easy and difficult conditions indicated no statistically significant group differences.

\section{Comparisons of percentage voxels activated in the ROls}

Univariate ANOVAs, comparing activation during the math task in the six ROIs (i.e., inferior frontal, middle frontal, superior frontal, superior parietal, supramarginal, and angular gyri) in the right and left hemisphere indicated no statistically significant differences between the VCFS and Control groups (Table 2). However, a nonsignificant statistical trend was noted in the left SMG; specifically, the percentage of voxels activated was higher among subjects with VCFS compared to controls $(\mathrm{F}=3.9$, $\mathrm{df}=1,14, P=0.068)$.

When comparing left SMG activation by task condition, a repeated-measures ANOVA model indicated a significant group difference in the pattern of activation during the "easy" and "difficult" conditions of the math task $(\mathrm{F}=8.7, \mathrm{df}=1,14$, $P=0.0106)$. Subjects with VCFS showed a distinct increase in activation during the "difficult" condition, whereas control subjects showed equivalent levels of activation during both conditions. ANOVA results comparing the mean percentage activation in the left SMG indicated significant group differences only during the "difficult" math task $(\mathrm{F}=7.8, \mathrm{df}=1,14$, $P=0.0141)$ and not during the "easy" math task $(\mathrm{F}=3.2, \mathrm{df}=$ $1,14, P=0.0941)$. No statistical differences were observed between groups in the right SMG during the "easy" or difficult" conditions of the math task. These results are shown in Table 3 .

\section{Voxel-based analyses}

Voxel-based analyses contrasting the "difficult" 3-operand computation with the control task pointed to two large clusters or regions that showed significantly more activation in subjects with VCFS than controls. The maximum difference was observed in the posterior left precentral gyrus $(Z$ - $\max =4.32, P$ $=0.000)$. Extending from this region, activation was observed at the juncture between the left lateral fissure (Brodman's Area (BA) 4/6) and the left SMG (BA 40) and in the left SMG. The second area of activation centered in the right insular gyri and parietal operculum $(\mathrm{Z}-\max =3.76, P=0.000)$ and extended into the right intraparietal sulcus and the right SMG. These data, including number of voxels in the clusters and the peak coordinates, are presented Table 4 . Figure 2, depicting the areas of activation, also shows a small cluster of increased activation in for the VCFS group in the right middle frontal gyrus. Voxel-based analysis did not demonstrate group differences for the "easy" computation task when compared to control stimuli. Further, there was no increase in brain activation in the controls compared to subjects with VCFS in either the "easy" or "difficult" math task.

\section{DISCUSSION}

Although preliminary because of small group sizes, the results of this first fMRI study of persons with VCFS suggest that affected individuals show abnormal brain activation during an arithmetic computation task. Thus, these findings serve as a

Table 1

Performance data collected during the math fMRI task

\begin{tabular}{|c|c|c|c|c|c|c|}
\hline & \multicolumn{2}{|c|}{$\operatorname{VCFS}(N=8)$} & \multicolumn{2}{|c|}{ Controls $(N=8)$} & \multirow{2}{*}{$\begin{array}{c}\mathrm{F} \\
\text { value }\end{array}$} & \multirow[b]{2}{*}{$P$ value } \\
\hline & Mean & SD & Mean & SD & & \\
\hline Total correct & $82.9 \%$ & 14.2 & $97.1 \%$ & 3.8 & 7.5 & 0.016 \\
\hline Easy correct & $90 \%$ & 14.3 & $99.2 \%$ & 2.4 & 3.2 & 0.094 \\
\hline Reaction time & 1538 & 161 & 1390 & 293 & 1.6 & 0.228 \\
\hline Reaction time & 2893 & 800 & 2490 & 685 & 1.2 & 0.298 \\
\hline
\end{tabular}

Mean performance scores (percentages) and reaction times (milliseconds) are shown for the total task (easy + difficult) and for the "easy" and "difficult" conditions of the task (epochs). 
Table 2

Mean percentage activation in frontal and parietal regions of interest during the fMRI math task

\begin{tabular}{|c|c|c|c|c|c|c|}
\hline Region of interest & Control $(N=8)$ & SD & $\operatorname{VCFS}(N=8)$ & $\mathrm{SD}$ & F values & $P$ values \\
\hline LIFG total math & 54.86 & 14.3 & 46.80 & 18.2 & 1.0 & 0.341 \\
\hline RIFG total math & 48.53 & 14.1 & 50.07 & 16.8 & 0.0 & 0.845 \\
\hline RMFG total math & 51.09 & 6.3 & 50.93 & 10.5 & 0.0 & 0.972 \\
\hline LSFG total math & 43.09 & 14.3 & 37.99 & 11.7 & 0.6 & 0.449 \\
\hline LSPL total math & 37.95 & 22.1 & 42.11 & 18.6 & 0.2 & 0.690 \\
\hline RSPL total math & 30.75 & 18.0 & 37.50 & 20.1 & 0.5 & 0.491 \\
\hline LSMG total math & 32.47 & 13.5 & 48.47 & 18.5 & 3.9 & 0.068 \\
\hline RSMG total math & 29.85 & 13.7 & 40.34 & 17.2 & 1.8 & 0.199 \\
\hline
\end{tabular}

L, left; R, right; IFG, inferior frontal gyrus; MFG, middle frontal gyrus; SFG, superior frontal gyrus; SPL, superior parietal lobe; SMG, supramarginal gyrus; ANG, angular gyrus.

Table 3

Mean percentage activation in the left supramarginal gyrus during the difficult math task and statistical comparisons of regions of interest activation between the VCFS and Control group

\begin{tabular}{|c|c|c|c|c|c|c|}
\hline Region of interest & Control $(N=8)$ & SD & $\operatorname{VCFS}(N=8)$ & $\mathrm{SD}$ & F values & $P$ values \\
\hline LSMG easy math & 42.40 & 12.0 & 33.96 & 12.5 & 1.9 & 0.190 \\
\hline RSMG easy math & 33.19 & 16.6 & 30.37 & 14.2 & 0.1 & 0.720 \\
\hline RSMG difficult math & 36.09 & 15.7 & 46.56 & 17.5 & 1.6 & 0.228 \\
\hline
\end{tabular}

For abbreviations, see Table 2.

Table 4

Brain areas that showed increased activation in subjects with VCFS $(N=8)$ compared to Control subjects $(N=8)$ during the difficult math task

\begin{tabular}{|c|c|c|c|c|c|c|}
\hline \multirow{2}{*}{$\frac{\text { Areas }}{\text { L Precentral gyrus (BA 4/6) }}$} & \multirow{2}{*}{$\frac{P \text { values }}{0.000}$} & \multirow{2}{*}{$\begin{array}{c}\begin{array}{c}\text { No. of } \\
\text { voxels in } \\
\text { cluster }\end{array} \\
6899\end{array}$} & \multirow{2}{*}{$\frac{\text { Z-max }}{4.32}$} & \multicolumn{3}{|c|}{$\begin{array}{l}\text { Peak } \\
\text { coordinates }\end{array}$} \\
\hline & & & & -28 & -24 & 46 \\
\hline $\begin{array}{l}\text { L Lateral fissure extending in the } \\
\text { LSMG (BA 40) }\end{array}$ & & & 3.89 & -58 & -30 & 10 \\
\hline LSMG (BA 40) & & & 3.76 & -48 & -505 & 50 \\
\hline $\begin{array}{l}\mathrm{R} \text { insular gyri and parietal } \\
\text { operculum }\end{array}$ & 0.000 & 5407 & 3.76 & 32 & -18 & 8 \\
\hline $\mathrm{R}$ intraparietal sulcus (BA 40) & & & 3.66 & 20 & -403 & 38 \\
\hline RSMG (BA 40) & & & 3.61 & 44 & -28 & 24 \\
\hline
\end{tabular}

For each significant cluster, region of activation, cluster level significance level, number of voxels activated, maximum $\mathrm{Z}$ score, and location of peak are shown.

potential link between previous observations of structural brain alterations in regions associated with mathematical reasoning ${ }^{17}$ and findings from behavioral research showing deficits in mathematical performance in VCFS. ${ }^{2,3,6,7}$
As hypothesized, children and adolescents with VCFS performed worse on an fMRI arithmetic task compared to typically developing comparison subjects. It is noteworthy that reaction times of subjects with VCFS during control trials were slower, yet not statistically different from the reaction times of the comparison group, suggesting that subjects with VCFS were not inhibited in their ability to attend and respond to the task.

In conjunction with decreased arithmetic performance, subjects with VCFS showed abnormal brain activation in regions of the parietal lobe, an area known to be involved in arithmetic processing. ${ }^{16,29}$ Specifically, unlike controls, a significant increase in neuronal activity in the left supramarginal gyrus was observed during the more difficult arithmetic problems in subjects with VCFS. This finding supports our a priori hypothesis that parietal lobe function is altered in subjects with VCFS and is consistent with a previous report of structural abnormalities in this region. ${ }^{17}$ Accordingly, alteration in parietal lobe morphology may contribute to decreased math performance commonly reported among children with VCFS. Other relative weaknesses in the neurocognitive profile of children with VCFS, including working memory difficulties and 


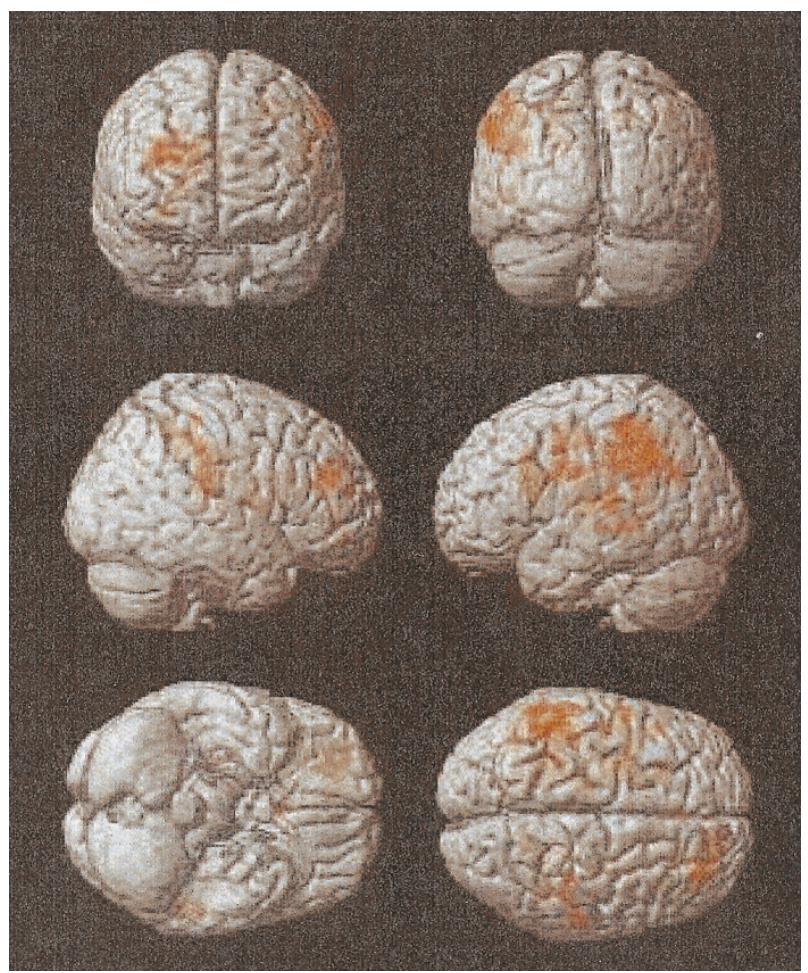

Fig. 2 Precise localization of increased brain activation during the difficult math task in the VCFS group $(N=8)$ when compared to control group $(N=8)$.

decreased visuospatial abilities, ${ }^{30}$ also could be due to alteration of parietal lobe structure and function.

Differences between subjects with VCFS and controls in left SMG activation were observed only during the more difficult (3-operand) mathematical reasoning condition. There are at least two possible explanations for this observation. First, high performance scores among the typically developing comparison group for both the hard and easy computational conditions suggest that the more difficult 3 -operand equations were not sufficiently demanding and, thus, did not require recruitment of additional neuronal resources to perform the task. While a harder task (e.g., multiplication and division, 2-digit numbers) may permit observation of a larger range of variation in brain activity and behavioral performance in controls, it also would likely preclude completion of the task by the subjects with VCFS. Second, the limited sample size might prevent detection of more discrete changes in regional recruitment among controls.

In contrast to the parietal lobe, no group differences in brain activation were observed for the frontal lobe using the ROIbased analysis. In a recent study of normal arithmetic processing using a similar experimental design, Menon et al. ${ }^{16} \mathrm{dem}-$ onstrated that both parietal and frontal lobe regions were involved in this cognitive function. In VCFS, levels of activity in frontal lobe subregions (IFG, MFG and SFG) were comparable with levels observed in controls, thereby suggesting that structural preservation of frontal gray and white matter observed in this condition ${ }^{17}$ may translate into relatively normal frontal lobe activation during mathematical computation.
Thus, our preliminary investigations into brain structure ${ }^{17}$ and function (this study) in VCFS are consistent in suggesting a dissociation between frontal and parietal lobe involvement in the neurocognitive features of affected individuals.

The voxel-based fMRI analyses, where no a priori hypotheses were utilized (see Fig. 2), provide further evidence and support for the ROI-based findings. In addition, the voxel-based findings point to other brain regions that may be involved in simple arithmetic computation. The observed activity in the right SMG and in a small cluster extending into the right frontal lobe is consistent with reports from other fMRI mathematical reasoning studies with normal subjects that have demonstrated increased activity in bilateral parietal and frontal/ prefrontal regions. ${ }^{14,16,31}$ Our observation of only right prefrontal activation may reflect a lack of brain lateralization or hemispheric specialization among persons with VCFS. Using fMRI, Burbaud et al. ${ }^{14}$ demonstrated leftward prefrontal lateralization of brain activity during mental calculation among normal right-handed subjects, whereas more bilateral prefrontal activation was observed in left-handed subjects.

An important limitation of the present study is the potential confounding effect of IQ. Overall differences in IQ between subjects with VCFS and controls could be responsible for some or all of the observed differences in brain activation. Children with lower IQ may need to recruit more resources to perform any cognitive task. However, if this was the case, one might expect subjects with VCFS to show increased activation in both frontal and parietal regions during mathematical reasoning. Future studies should include a control group composed of children with comparable IQ levels in order to discern whether differences in brain activation are due to VCFS or to a general decrease in cognitive ability.

In summary, we have presented a preliminary study that is the first to explore brain function in VCFS. Potential directions for future imaging studies of this condition include a finer subparcellation of the parietal lobe, and investigation of other parietal-based cognitive functions that are impaired in VCFS (e.g., working memory). Most importantly, use of an IQmatched, developmentally disabled control group would allow measurement of the specific effect of VCFS on neuronal activity, independent of general cognitive ability. Our results suggest that investigations using recently developed neuroimaging tools will provide new insights into the neural mechanisms underlying cognitive deficits in VCFS and other neurodevelopmental disorders.

\section{Acknowledgment}

The research presented in this manuscript was supported by the National Swiss Research Fund to Dr. Stephan Eliez and NIH grants MH01142 and HD31715 to Dr. Allan Reiss. This work also was partially supported by grants from the M.I.N.D. Institute and the Packard Foundation to Dr. Reiss.

\section{References}

1. Gerdes M, Solot C, Wang PP, Moss E, LaRossa D, Randall P, Goldmuntz E, Clark BJ, 3rd, Driscoll DA, Jawad A, Emanuel BS, McDonald-McGinn DM, Batshaw ML, 
Zackai EH. Cognitive and behavior profile of preschool children with chromosome 22q11.2 deletion. Am J Med Genet 1999;85:127-133.

2. Golding-Kushner KJ, Weller G, Shprintzen RJ. Velo-cardio-facial syndrome: language and psychological profiles. J Craniofac Genet Dev Biol 1985;5:259-266.

3. Moss EM, Batshaw ML, Solot CB, Gerdes M, McDonald-McGinn DM, Driscoll DA, Emanuel BS, Zackai EH, Wang PP. Psychoeducational profile of the 22q11.2 microdeletion: a complex pattern. J Pediatr 1999;134:193-198.

4. Swillen A, Devriendt K, Legius E, Eyskens B, Dumoulin M, Gewillig M, Fryns JP. Intelligence and psychosocial adjustment in velocardiofacial syndrome: a study of 37 children and adolescents with VCFS. J Med Genet 1997;34:453-458.

5. Swillen A, Devriendt K, Legius E, Prinzie P, Vogels A, Ghesquiere P, Fryns JP. The behavioural phenotype in velo-cardio-facial syndrome (VCFS): from infancy to adolescence. Genet Couns 1999;10:79-88.

6. Kok LL, Solman RT. Velocardiofacial syndrome: learning difficulties and intervention. J Med Genet 1995;32:612-618.

7. Swillen A, Vandeputte L, Cracco J, Maes B, Ghesquire P, Devriendt K, Fryns J-P. Neuropsychological, learning, and psychological profile of primary school aged children with the velo-cardio-facial syndrome (22q11 deletion): evidence for a nonverbal learning disability? Child Neuropsychol 1999;5:230-241.

8. Benson DF, Weir WF. Acalculia: acquired anarithmetia. Cortex 1972;8:465-472.

9. Henschen S. Klinische und anatomische beitraege sur pathologie des Gehirns. Vol 5. Stockholm: Nordiska Bokhandeln, 1920.

10. McCarthy RA, Warrington EK. Evidence for modality-specific meaning systems in the brain. Nature 1988;334:428-430.

11. Takayama Y, Sugishita M, Akiguchi I, Kimura J. Isolated acalculia due to left parietal lesion. Arch Neurol 1994;51:286-291.

12. Warrington EK. The fractionation of arithmetical skills: a single case study. QJ Exp Psychol A 1982;34:31-51.

13. Whalen J, McCloskey M, Lesser RP, Gorden B. Localizing arithmetic processes in the brain: evidence from a transient deficit during cortical stimulation. J Cogn Neurosci 1997;9:409-417.

14. Burbaud P, Degreze P, Lafon P, Franconi JM, Bouligand B, Bioulac B, Caille JM, Allard M. Lateralization of prefrontal activation during internal mental calculation: a functional magnetic resonance imaging study. J Neurophysiol 1995;74:2194-200.

15. Dehaene S, Spelke E, Pinel P, Stanescu R, Tsivkin S. Sources of mathematical thinking: behavioral and brain-imaging evidence. Science 1999;284:970-974.

16. Menon V, Rivera SM, White CD, Glover GH, Reiss AL. Dissociating prefrontal and parietal cortex activation during arithmetic processing. Neuroimage 2000;12:357365 .
17. Eliez S, Schmitt JE, White CD, Reiss AL. Children and adolescents with velocardiofacial syndrome: a volumetric MRI study. Am J Psychiatry 2000;157:409-415.

18. Shaikh TH, Kurahashi H, Saitta SC, O'Hare AM, Hu P, Roe BA, Driscoll DA, McDonald-McGinn DM, Zackai EH, Budarf ML, Emanuel BS. Chromosome 22-specific low copy repeats and the 22q11.2 deletion syndrome: genomic organization and deletion endpoint analysis. Hum Mol Genet 2000;9:489-501.

19. Karayiorgou M, Morris MA, Morrow B, Shprintzen RJ, Goldberg R, Borrow J, Gos A, Nestadt G, Wolyniec PS, Lasseter VK, et al. Schizophrenia susceptibility associated with interstitial deletions of chromosome 22q11. Proc Natl Acad Sci USA 1995; 92:7612-7616.

20. Kurahashi H, Tsuda E, Kohama R, Nakayama T, Masuno M, Imaizumi K, Kamiya T, Sano T, Okada S, Nishisho I. Another critical region for deletion of 22q11: a study of 100 patients. Am J Med Genet 1997;72:180-185.

21. Hayes C, Mathias C. Improved brain coil for fMRI and high resolution imaging. Proceedings of the 4th Annual Meeting of the ISMRM. New York; 1996:1414.

22. Glover GH, Lai S. Self-navigated spiral fMRI: interleaved versus single-shot. Magn Reson Med 1998;39:361-368.

23. Cohen JD, MacWhinney B, Flatt M, Provost J. PsyScope. A new graphic interactive environment for designing psychology experiments. Behav Res Methods Instrum Comput 1993;25:257-271.

24. Talairach J, Tournoux P. Co-planar stereotaxic atlas of the human brain. Stuttgart, New York: Georg Thieme Verlag, 1988.

25. Holmes AP, Friston KJ. Generalisability, random effects \& population inference. Neuroimage 1998;7:S754.

26. Poline JB, Worsley KJ, Evans AC, Friston KJ. Combining spatial extent and peak intensity to test for activations in functional imaging. Neuroimage 1997;5:83-96.

27. Duvernoy HM, Bourgouin P. The human brain : surface, three-dimensional sectional anatomy with MRI, and blood supply. Wien; New York: Springer, 1999.

28. Ono J, Harada K, Mano T, Sakurai K, Okada S. Differentiation of dys- and demyelination using diffusional anisotropy. Pediatr Neurol 1997;16:63-66.

29. Menon V, Rivera SM, White CD, Eliez S, Glover GH, Reiss AL. Functional optimzation of arithmetic processing in perfect performers. Cogn Brain Res 2000;9:343-345.

30. Wang PP, Woodin MF, Kreps-Falk R, Moss EM. Research on behavioral phenotypes: velocardiofacial syndrome (deletion 22q11.2). Dev Med Child Neurol 2000; 42:422-42s7.

31. Rueckert L, Lange N, Partiot A, Appollonio I, Litvan I, Le Bihan D, Grafman J. Visualizing cortical activation during mental calculation with functional MRI. Neuroimage 1996;3:97-103. 